\title{
Evaluation of the Accuracy of a New Cuffless Magnetoplethysmography Blood Pressure Monitor in Hypertensive Patients
}

\author{
Sua Kim ${ }^{a}$ Jung Dong Lee ${ }^{b}$ Jeong Bae Park ${ }^{b}$ Seungjin Jang ${ }^{c}$ \\ Jungchae Kim ${ }^{d}$ Sang-Suk Lee ${ }^{e}$ \\ a Department of Critical Care Medicine, Samsung Medical Center, Sungkyunkwan University, \\ bJB Lab and Clinic, 'Intelligence Lab, Convergence Center, LG Electronics, and dArtificial \\ Intelligence Lab, SW Center, LG Electronics, Seoul, and e Department of Oriental Biomedical \\ Engineering, Sangji University, Wonju, Republic of Korea
}

\section{Keywords}

Blood pressure measurement - Cuffless device - Magnetoplethysmography sensor . Hypertension

\begin{abstract}
Objective: There is a growing demand for cuffless blood pressure (BP) measurement as an easy alternative to cuff-occlusion-based BP measurement. We assessed the accuracy of a new cuffless, watch-style BP monitor with a magnetoplethysmography (MPG) sensor compared to two standard auscultatory and oscillatory BP monitors. Subjects and Methods: A total of 34 patients with uncontrolled hypertension (systolic $B P \geq 150 \mathrm{~mm} \mathrm{Hg}$ or diastolic $B P \geq 95 \mathrm{~mm} \mathrm{Hg}$ ) were enrolled in the study. BP was measured by two conventional monitors and the new device during the pre-exercise phase, during isometric handgrip exercise, and during the recovery phase ( $5 \mathrm{~min}$ after exercise). The correlation between monitors was assessed using intraclass correlation coefficient (ICC) and Bland-Altman plots. Results: Although two reference monitors produced highly correlated BP measurements, each was differentially correlated with BP measurements obtained by the new MPG monitor. During exercise, the mean difference between systolic BP obtained by the MPG and oscillatory monitors was $>7 \mathrm{~mm} \mathrm{Hg}$ with an ICC of $0.549(95 \% \mathrm{Cl} 0.264-0.746)$ in systole and 0.737 (95\% $\mathrm{Cl} 0.534-0.859)$ in diastole. The ICC between the auscultatory monitor and the MPG monitor was 0.753 ( $95 \% \mathrm{Cl} 0.559-0.868$ ) in systole and $0.841(95 \% \mathrm{Cl} 0.706-0.918)$ in diastole after exercise. Bland-Altman plots also indicated that the performance of the new MPG device was very similar to that of the auscultatory monitor. Conclusion: Although the performance of the new MPG monitor was comparable to that of the reference monitors used in this study, improved stability and accuracy are necessary for accurate BP evaluation during dynamic activity.


Kim et al.: Evaluation of the Accuracy of a New Cuffless Magnetoplethysmography Blood Pressure Monitor in Hypertensive Patients

\section{Introduction}

The aging population is rapidly growing in today's society due to important medical advances that have vastly prolonged life expectancy. As people live longer, the prevalence of degenerative diseases is also on the rise. Hypertension is one of the most prevalent yet preventable degenerative diseases facing the elderly and a major contributor to mortality. Although several therapeutic agents have been developed and have indeed improved health outcomes for many [1-3], the prevalence of hypertension continues to increase [4], and in many cases hypertension is not properly controlled [5]. This represents a critical public health care issues, as hypertension, if not properly controlled, can lead to myocardial infarction, stroke, renal failure, and even death. Precise blood pressure (BP) monitoring and control are critical, not only for early diagnosis, but also for keeping hypertension in check. Diagnosis as well as proper administration of antihypertensive agents require accurate BP measurement. BP depends on an individual's psychological and physical condition and is constantly changing. Because of this, BP measured during a narrow time window at the doctor's office, or in the clinic or hospital, is not regarded as a concrete standard [6-8]. Ambulatory and home BP monitoring [9-11] provide a more complete picture of an individual's BP range, and such measurements are actively used in clinical settings for diagnosis and treatment. Ambulatory and home BP monitoring are especially recommended for patients suspected to suffer from white-coat hypertension or masked hypertension $[12,13]$. When BP is not well controlled, it becomes especially critical to cross-compare office BP measurements to those of home BP measurements; however, even if BP seems properly controlled, patients with a nondipping BP pattern are likely to have worse prognoses than those with a dipping BP pattern, further emphasizing the importance of precise home BP monitoring.

Despite their importance in effective hypertension monitoring and control, home and ambulatory BP measurements suffer from inconvenience, and home BP measurement is often inaccurate $[14,15]$. Comfort is also a significant concern. Because of this, home and ambulatory BP monitors suffer from biases, introduced both by the physician and the patient. Both patients and clinicians would benefit greatly from a convenient, accurate BP monitor, which could prove vital for improved, successful antihypertensive therapeutic approaches.

There have been many attempts to develop a BP measuring device meeting the needs of both patients and physicians [16]. A portable cuffless device that a patient can wear like a watch is currently under development. The watch-like design increases comfort, both through a low profile and by reducing discomfort often associated with cuff BP measurement devices. It is also more convenient for a patient, as it can measure BP irrespective of a patient's physical position by utilizing a magnetoplethysmography (MPG) sensor. This negates the need for a patient to identify a good time during the day every day when he or she can stop engaging in normal daily activities and remain in the required position for accurate BP measurement, which in turn leads to more consistent BP monitoring. Here we assess the accuracy, and therefore validity, of this new cuffless BP monitor. We used the monitor to measure BP both during and after exercise, and additionally used oscillometric and auscultatory sphygmomanometers as validated references for cross-comparison. Our results suggest that this new device is highly accurate, and we suggest that this device can be used to facilitate comfortable, convenient ambulatory and home BP monitoring. 
Kim et al.: Evaluation of the Accuracy of a New Cuffless Magnetoplethysmography Blood Pressure Monitor in Hypertensive Patients

Fig. 1. Cross-sectional diagram illustrating the structure of the new magnetoplethysmography sensor-based cuffless blood pressure monitor.

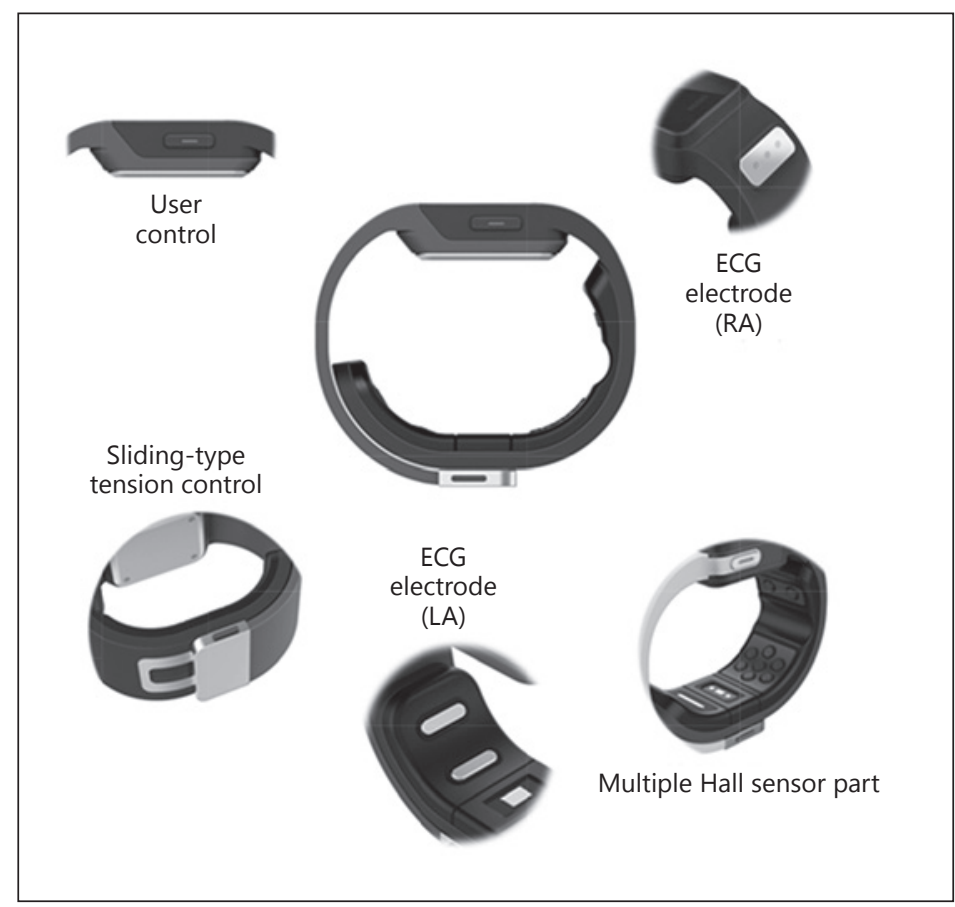

\section{Methods}

\section{Study Population}

Patients diagnosed with hypertension aged between 18 and 65 years were screened for inclusion in our study, where patients were recruited in Cheil General Hospital, Republic of Korea. Participating patients were required to have either systolic $\mathrm{BP} \geq 150 \mathrm{~mm} \mathrm{Hg}$ or diastolic $\mathrm{BP} \geq 95 \mathrm{~mm} \mathrm{Hg}$, and we did not take into consideration medical intervention for BP control. Since a key component of our study was an isometric handgrip exercise, we excluded patients with visual disturbance, history of musculoskeletal disease, and heart failure or other structural heart disease, which would have made participation in the handgrip exercise too dangerous. A total of 34 patients who fit the inclusion criteria and provided informed consent were included in the study. Baseline (pre-exercise) BP was determined for patients, after which they were asked to engage in a handgrip exercise, during which BP was measured once again. BP was measured a third time 5 min after cessation of activity.

\section{Isometric Handgrip Exercise}

As described above, patients performed an isometric handgrip exercise during the study visit. We included this activity to test the capabilities of the new MPG device. We performed a pretest evaluation of the maximal handgrip strength. Three measurements were collected at 30-s intervals and the highest value of the three was regarded as the maximal handgrip strength. Patients wore both the HEM-7121 monitor and the new MPG monitor on their left arm while performing the isometric handgrip exercise with their right hand. During the handgrip exercise, patients maintained their grip at 30\% of the maximal grip strength for 2-3 min while BP measurements were collected.

\section{The MPG Device}

The new BP monitor evaluated in our study is a magnetoplethysmograph utilizing a Hall sensor and is worn like a watch (Fig. 1). The device has nine permanent magnets embedded in a wristband, which is designed to sit in very close proximity to the radial artery by adjusting the band. When blood flows through the artery and pulses with each heartbeat, the magnets move by BP transmitted to the skin; BP is estimated by a morphological index of pulse waves derived by the movement of the magnets. To be specific, the device is first calibrated using the index derived by baseline BP and corresponding pulse waves. Then, by analyzing changed forms of pulse waves, the device estimates BP. The device is Bluetooth-enabled and is designed to 
Kim et al.: Evaluation of the Accuracy of a New Cuffless Magnetoplethysmography Blood Pressure Monitor in Hypertensive Patients

Table 1. Demographics of the 34 patients

$\begin{array}{lc}\text { Age, years } & 52.82 \pm 15.62 \\ \text { Females } & 22(64.71 \%) \\ \text { Males } & 12(35.29 \%) \\ \text { Body mass index } & 26.78 \pm 4.49 \\ \text { Height, cm } & 163.88 \pm 9.69 \\ \text { Weight, kg } & 72.24 \pm 15.57 \\ \text { Patients with diabetes mellitus } & 10(29.41 \%) \\ \text { Patients with hypertension } & 32(94.12 \%)\end{array}$

Values are presented as mean \pm standard deviation or $\mathrm{n}(\%)$

be used with a smartphone. BP measurement is initiated via an app, which also receives the BP readout from the monitor. When monitoring BP using this device, we obtained a total of 2-10 measurements, collecting a total of 249 measurements from the 34 patients included in the study.

\section{Auscultatory and Oculometric BP Monitors}

We used two different sphygmomanometers as reference monitors in this study. For auscultatory BP measurements, we utilized the Accoson Greenlight 300 (AC Cossor \& Son [Surgical] Ltd., London, UK), an electronic and manual nonautomated mercury-free device; for oscillometric BP measurements, we utilized the Omron HEM-7121 (Omron Healthcare Co. Ltd., Tokyo, Japan), an automated device. Both devices utilize cuffs to measure BP; for this study, all patients wore the cuffs on the left upper arm at heart height while seated. As described above, BP was measured three times for each patient: before, during, and after exercise. The Greenlight 300 and the Omron HEM-7121 were used to measure BP before exercise. During exercise, the new monitor and the Omron HEM-7121 were used, and after exercise, the new device and the Greenlight 300 were used.

\section{Statistical Analysis}

All results are expressed as mean \pm standard deviation and median (interquartile range) for continuous variables and as percentage for categorical variables. We evaluated the accuracy of the new MPG device as compared to the reference BP monitors by computing the intraclass correlation coefficient (ICC) and building a Bland-Altman plot. ICC values were interpreted as follows: $>0.75$ was excellent, $0.40-0.75$ was fair to good, and $<0.40$ was poor [17]. A $p$ value $<0.05$ was considered statistically significant. Database management and statistical analyses were performed using Statistical Analysis System (SAS), version 9.3 (SAS Institute, Cary, NC, USA).

\section{Results}

A total of 34 patients meeting our inclusion criteria were included in this study. The mean age was 52.8 years, and $64.7 \%$ of the subjects were women. Among them, 29.4\% were diagnosed with diabetes. All patient demographics are summarized in Table 1.

Using the Omron HEM-7121 monitor, the average systolic BP across the entire study cohort was $159.7 \pm 14.7 \mathrm{~mm} \mathrm{Hg}$ (range 131.0-192.0 mm Hg); the average diastolic BP was $96.9 \pm 11.8 \mathrm{~mm} \mathrm{Hg}$ (range 77.0-124.0 mm Hg). The Greenlight 300 yielded nearly identical results, with a systolic BP of $159.8 \pm 14.4 \mathrm{~mm} \mathrm{Hg}$ and an average diastolic BP of $98.3 \pm 12.0$ $\mathrm{mm} \mathrm{Hg}$. Indeed, the correlation coefficient of systolic and diastolic BP measured by the two devices was significant ( $r=0.845, p=0.0002$ and $r=0.882, p=0.0791$, respectively). Similarly, the ICC for systolic and diastolic BP was 0.840 (95\% CI 0.703-0.917) and 0.882 (95\% CI 0.777-0.939), respectively (Table 2).

To determine the performance of the new MPG device, patients were also asked to exercise by performing an isometric handgrip exercise, and BP was measured both during 
Kim et al.: Evaluation of the Accuracy of a New Cuffless Magnetoplethysmography Blood Pressure Monitor in Hypertensive Patients

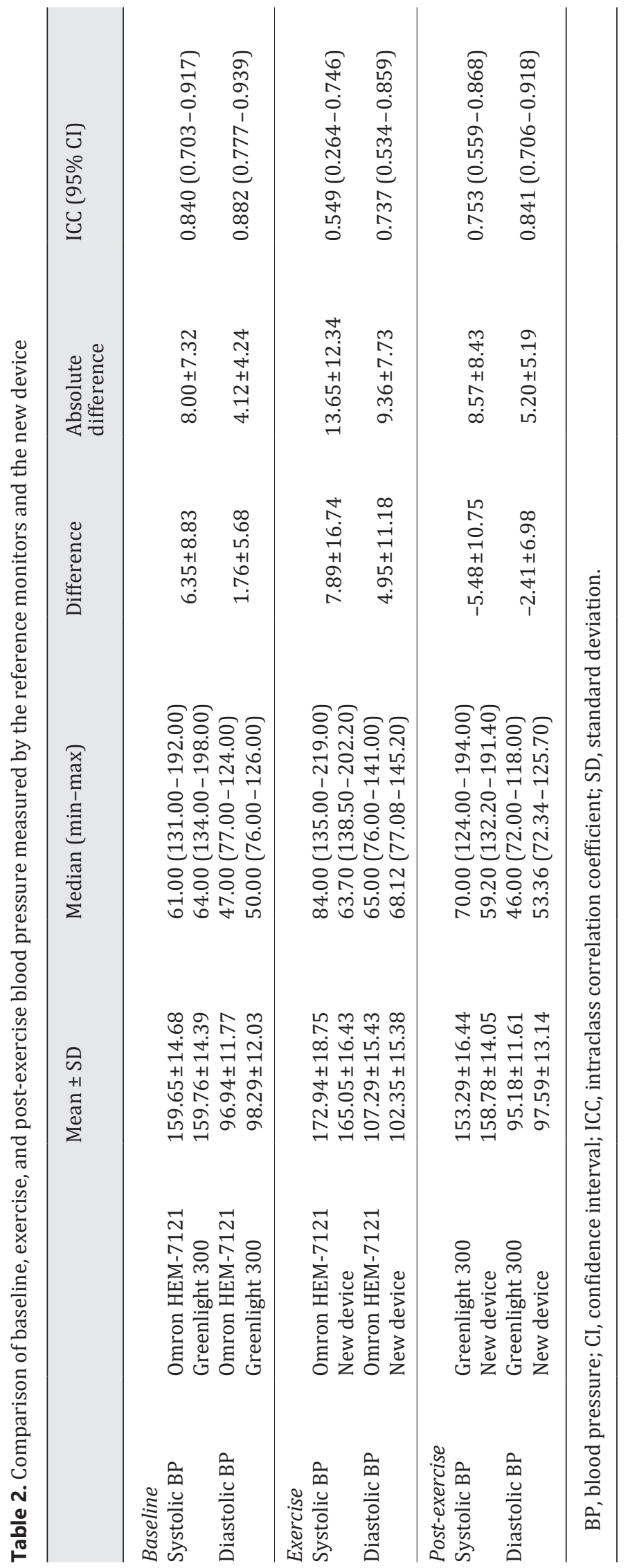




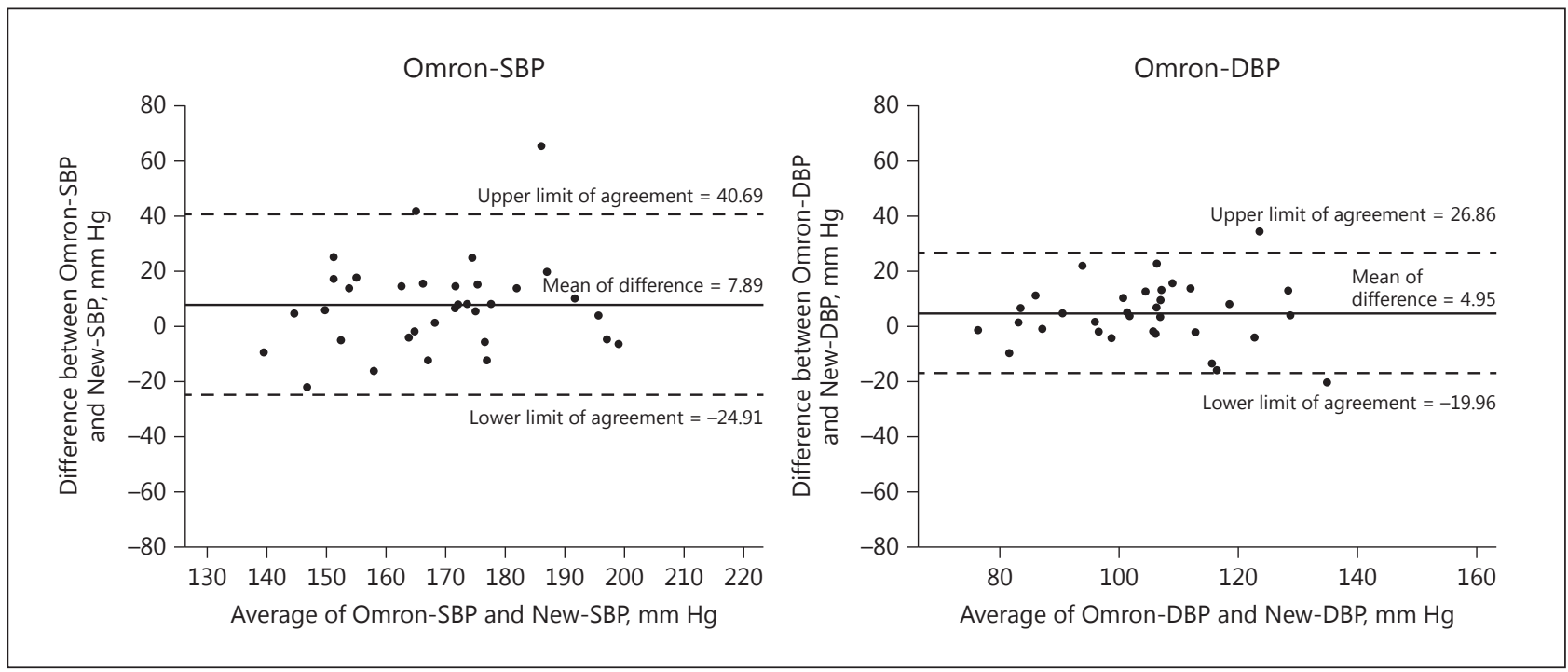

Fig. 2. Bland-Altman plots of systolic and diastolic blood pressure values obtained by the Omron HEM-7121 blood pressure monitor and the new MPG monitor during exercise. It shows good agreement between the Omron HEM-7121 and MPG monitors. SBP, systolic blood pressure; DBP, diastolic blood pressure.

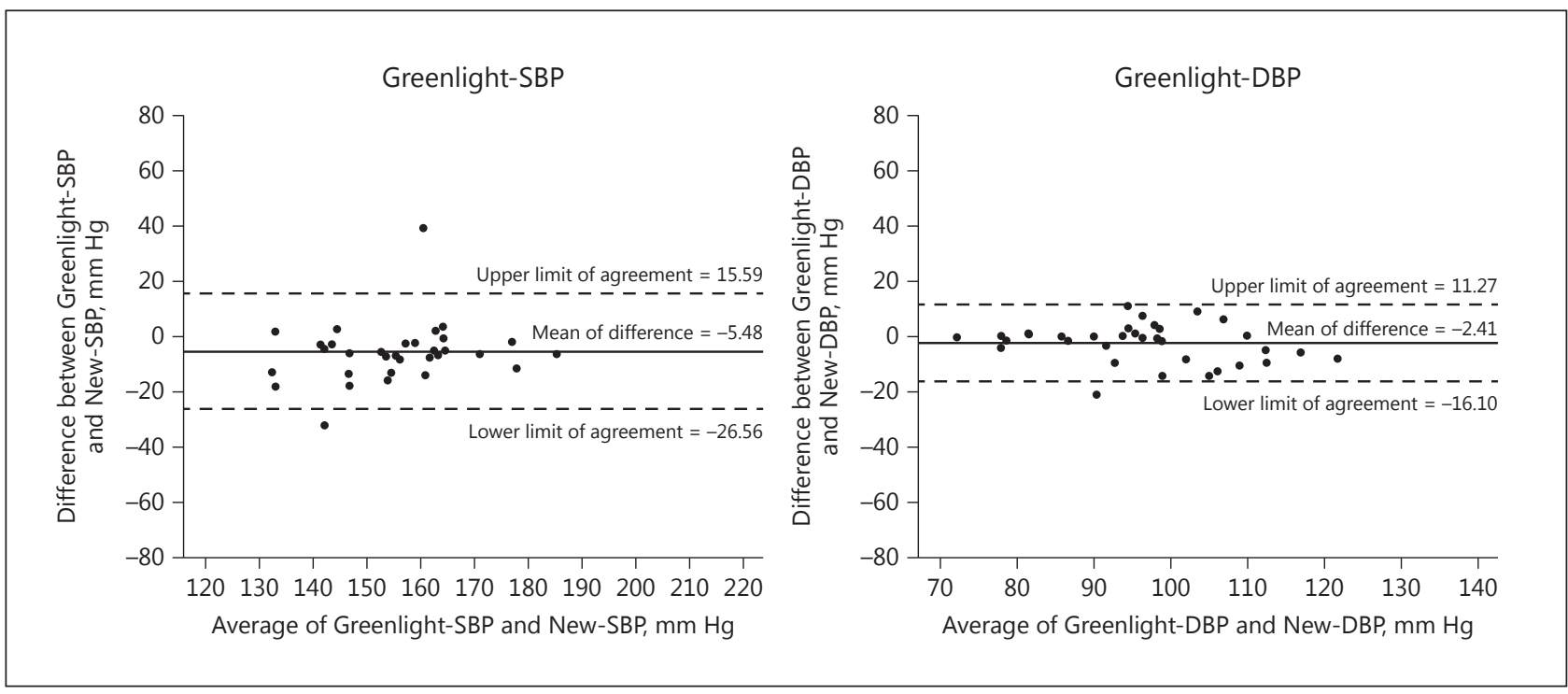

Fig. 3. Bland-Altman plots of systolic and diastolic blood pressure values obtained by the Greenlight 300 blood pressure monitor and the new MPG monitor after exercise. The Greenlight 300 and the MPG monitors agreed well, and their agreement was better than that between the Omron HEM-7121 and MPG monitors. SBP, systolic blood pressure; DBP, diastolic blood pressure.

exercise and 5 min after cessation of exercise. During exercise, the average systolic and diastolic BP as measured by the Omron HEM-7121 monitor increased to $172.9 \pm 18.8 \mathrm{~mm} \mathrm{Hg}$ and $107.3 \pm 15.4 \mathrm{~mm} \mathrm{Hg}$, respectively. The same measurements obtained by the new device were lower than those of the two reference devices, with an average systolic BP of $165.1 \pm$ $16.4 \mathrm{~mm} \mathrm{Hg}$ and an average diastolic BP of $102.4 \pm 15.4 \mathrm{~mm} \mathrm{Hg}$. Using the Greenlight 300 
Kim et al.: Evaluation of the Accuracy of a New Cuffless Magnetoplethysmography Blood Pressure Monitor in Hypertensive Patients

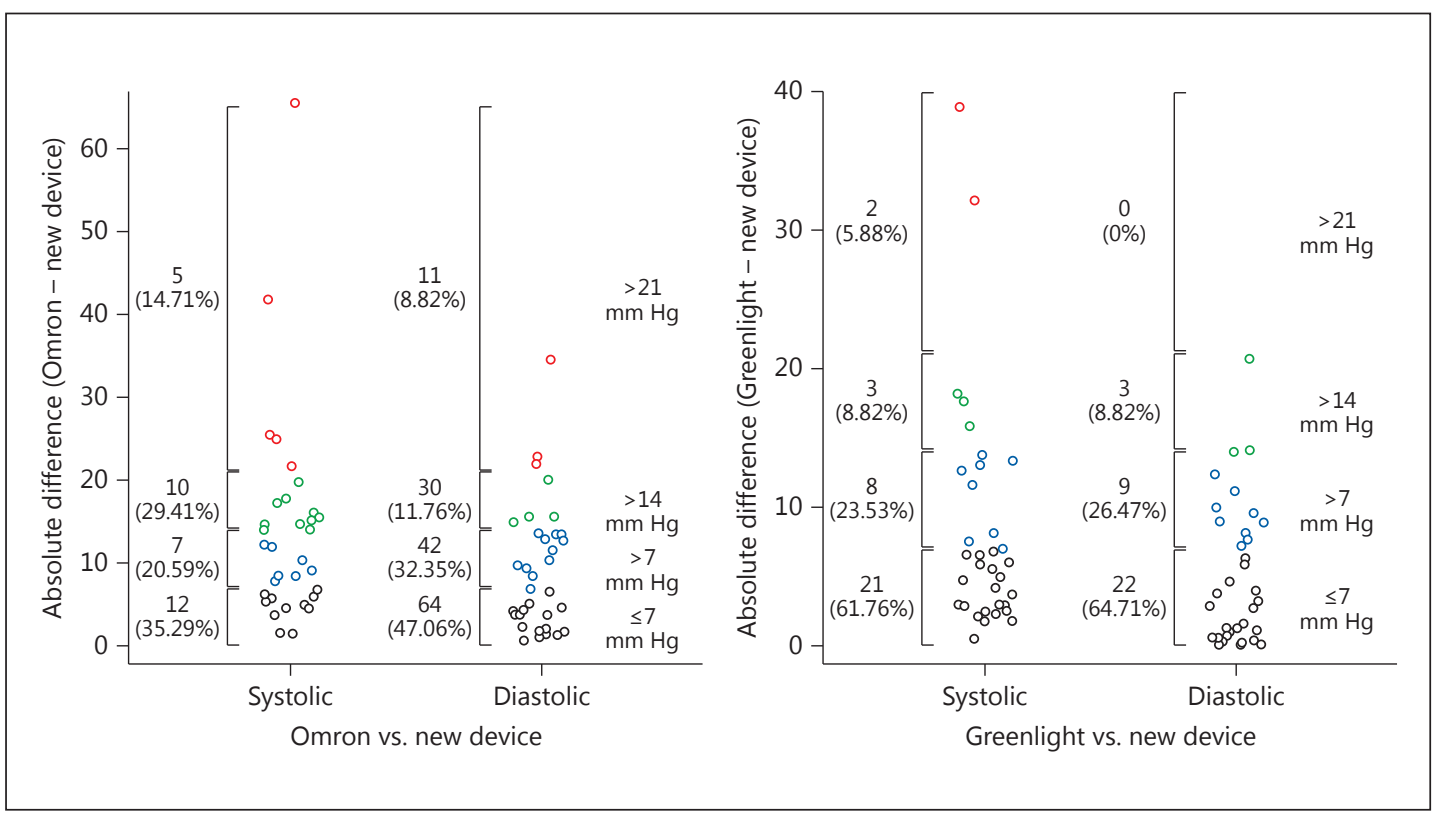

Fig. 4. Scatterplots of patients by absolute blood pressure differences obtained with the reference blood pressure monitors and the new MPG monitor. When exercising, this percentage of blood pressure difference $<7 \mathrm{~mm} \mathrm{Hg}$ between the two devices was $35.3 \%$ in systole and $47.1 \%$ in diastole (left). After exercise (resting), this percentage was $61.8 \%$ in systole and $64.7 \%$ in diastole (right).

monitor, the average post-exercise systolic BP was $153.3 \pm 16.4 \mathrm{~mm} \mathrm{Hg}$ and the average postexercise diastolic BP $95.2 \pm 11.6 \mathrm{~mm} \mathrm{Hg}$. In contrast to our observations when comparing the new MPG monitor and the Omron HEM-7121 monitor, the average BP was slightly higher with the MPG monitor, at $158.8 \pm 14.1 \mathrm{~mm} \mathrm{Hg}$ for systolic BP and $97.6 \pm 13.1 \mathrm{~mm} \mathrm{Hg}$ for diastolic BP (Table 2).

We next used Bland-Altman plots (Fig. 2) and calculated the ICC to determine whether BP measurements between the reference monitors and the MPG monitor were correlated. The average difference in systolic BP obtained by the Omron HEM-7121 and MPG monitors during exercise was $13.7 \pm 12.3 \mathrm{~mm} \mathrm{Hg}$ with an ICC of 0.549 (95\% CI 0.264-0.746). The correlation for diastolic BP was better, with an average difference of $9.4 \pm 7.7 \mathrm{~mm} \mathrm{Hg}$ and an ICC of 0.737 (95\% CI 0.534-0.859) (Table 2).

The correlation between post-exercise BP measurements obtained by the Greenlight 300 and MPG monitors was higher than that observed between the Omron HEM-7121 and MPG monitors. The average difference in systolic BP obtained by the two monitors was $8.6 \pm$ $8.4 \mathrm{~mm} \mathrm{Hg}$ with an ICC of 0.753 (95\% CI 0.559-0.868), while the average difference in diastolic $\mathrm{BP}$ was $5.2 \pm 5.2 \mathrm{~mm} \mathrm{Hg}$, with an ICC of 0.841 (95\% CI 0.706-0.918). The Bland-Altman plots supported these results, indicating higher agreement between the Greenlight 300 and the MPG monitors than between the Omron HEM-7121 and MPG monitors (Fig. 2, 3).

We additionally quantified the percentage of patients for whom the difference in BP obtained between the reference monitors and the new monitor was $<7 \mathrm{~mm} \mathrm{Hg}$. When exercising, this percentage was $35.3 \%$ in systole and $47.1 \%$ in diastole. After exercise, this percentage was $61.8 \%$ in systole and $64.7 \%$ in diastole (Fig. 4). We also quantified the percentage of patients for whom the difference in BP obtained between the reference and new monitors was $>21 \mathrm{~mm} \mathrm{Hg}$. This percentage was $14.7 \%$ in systole and $8.8 \%$ in diastole during exercise and $5.9 \%$ in systole and $0.0 \%$ in diastole after exercise (Fig. 4). 
Kim et al.: Evaluation of the Accuracy of a New Cuffless Magnetoplethysmography Blood Pressure Monitor in Hypertensive Patients

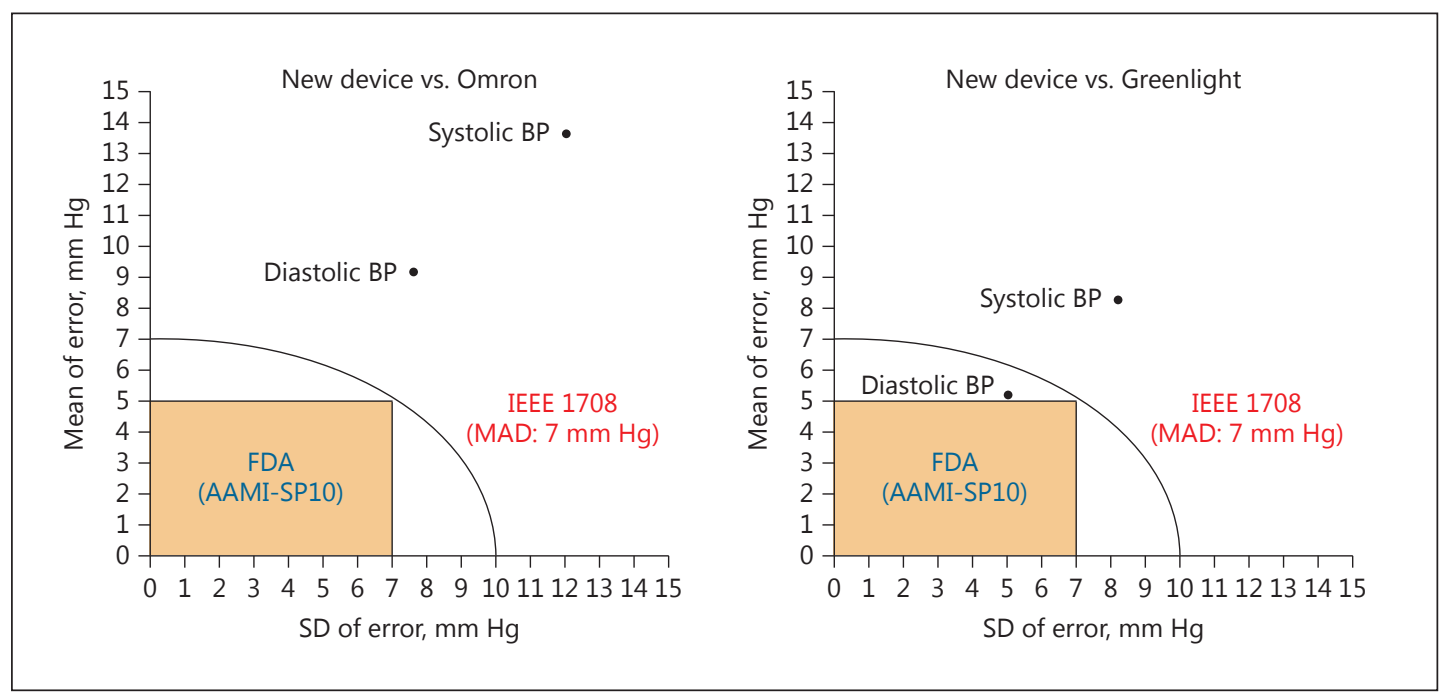

Fig. 5. Mean and standard deviation of the absolute blood pressure (BP) differences between the reference BP monitors and the new MPG monitor according to the FDA's standard (absolute BP differences $\leq 5 \pm 8 \mathrm{~mm}$ $\mathrm{Hg}$ ) and the IEEE 1708's standard (mean absolute BP difference $<7 \mathrm{~mm} \mathrm{Hg}$ ). SD, standard deviation; MAD, mean absolute difference.

\section{Discussion}

For proper hypertension diagnosis and control, it is critical to obtain accurate, precise BP measurements. Home BP measurements are especially useful as they provide a more complete picture of a patient's normal BP range. However, home monitors suffer from inconvenience, and patients often do not understand why home measurements are critical to their care. Even when patients are adequately educated, they are often unable to measure their BP appropriately. Although ambulatory BP monitoring has been proposed as a solution, it also suffers from inconvenience and therefore only partially solves the problem. Therefore, the development of convenient, easy-to-wear, and easy-to-use BP monitors could solve a critical healthcare issue, and many groups have attempted to develop such a device $[16,18]$. Additionally, the Institute of Electrical and Electronics Engineers (IEEE) has suggested a standard for wearable, cuffless BP measuring devices [19] for more accurate BP evaluation.

The new MPG device evaluated here effectively meets all of the criteria for improved home BP monitors. Patients wear it like a watch, which is both more comfortable and convenient than existing home and ambulatory monitors. Additionally, the device can be used in conjunction with a smartphone application, streamlining both usage and data collection and facilitating telemonitoring, which enables physicians to more closely monitor patient status. This is particularly significant given that real-time BP monitoring may improve the prognosis [20] for patients suffering from atypical conditions such as pregnancy-induced hypertension.

To fulfill the standards for evaluating new BP monitors suggested by the IEEE, we enrolled at least 25 hypertensive patients between 18 and 65 years of age and evaluated BP over a dynamic range. We compared the new monitor with standardized, conventional auscultatory and oscillatory BP monitors which we confirmed produced highly correlated BP measurements. The new monitor yielded BP measurements similar to the auscultatory monitor, but not the oscillatory monitor, especially after exercise; however, the mean absolute difference between the reference monitors and the new monitor was $>7 \mathrm{~mm} \mathrm{Hg}$, the standard suggested by the IEEE (Fig. 5). Because of this, commercialization of the device for ambulatory BP moni- 
Kim et al.: Evaluation of the Accuracy of a New Cuffless Magnetoplethysmography Blood Pressure Monitor in Hypertensive Patients

toring may be difficult. Nevertheless, the ICC and Bland-Altman plots suggested that the monitor performed well in comparison to the reference monitors. Therefore, we feel that the data we present here will prove useful for improving the accuracy of the device.

There are several limitations to this study. First, we only evaluated the monitors in hypertensive patients, not in normal, healthy individuals. Additionally, we did not compare the new device to both reference monitors at all three time points for which BP was measured. This is important because although the reference monitors yielded highly correlated BP measurements, the new device performed more similarly to the Greenlight 300. The difference may be due to the higher range of BP measured during exercise as opposed to before or after, but it is impossible to determine whether this is the case with the current data. Additionally, we could not evaluate BP using the reference monitors and the new device simultaneously because BP cannot be measured from the ipsilateral radial artery while a cuff-type monitor is in use. Instead, we asked individuals to maintain an even handgrip power for 2-3 min and confirmed their success in doing so using a dynamometer monitor. This enabled us to measure $\mathrm{BP}$ with the two devices separately; however, it is possible that immeasurable differences in grip strength were associated with measurable differences in BP and are responsible for the differences observed between the reference monitors and the new device. Finally, we evaluated BP in a controlled clinical environment, which is not likely to be reflective of the environment in which patients will measure ambulatory BP. This should be assessed after the final product design is complete, as any simple change may highly influence accuracy.

\section{Conclusions}

Precise and accurate home BP measurement is critical for effective BP monitoring. Such measurements not only enable proper $\mathrm{BP}$ control and facilitate accurate prognosis prediction, but also improve patient compliance. Here we describe a new, cuffless MPG device. Our results, comparing this device to two standardized monitors, suggest that although this new device is promising, further studies are needed to improve the accuracy of the device. In the future, it is likely to prove an effective tool for improved home BP monitoring.

\section{Acknowledgments}

This study was sponsored by LG Electronics, Republic of Korea.

\section{Statement of Ethics}

The study was approved by the Institutional Review Board of Cheil General Hospital, and all patients provided written informed consent.

\section{References}

1 Staessen JA, Fagard R, Thijs L, et al: Randomised double-blind comparison of placebo and active treatment for older patients with isolated systolic hypertension. The Systolic Hypertension in Europe (Syst-Eur) Trial Investigators. Lancet 1997;350:757-764.

2 Beckett NS, Peters R, Fletcher AE, et al: Treatment of hypertension in patients 80 years of age or older. N Engl J Med 2008;358:1887-1898.

3 Prevention of stroke by antihypertensive drug treatment in older persons with isolated systolic hypertension. Final results of the Systolic Hypertension in the Elderly Program (SHEP). SHEP Cooperative Research Group. JAMA 1991;265:3255-3264. 
Kim et al.: Evaluation of the Accuracy of a New Cuffless Magnetoplethysmography Blood Pressure Monitor in Hypertensive Patients

4 Wikimedia Commons: Life expectancy at birth by region 1950-2050. https://commons.wikimedia.org/wiki/ File:Life_Expectancy_at_Birth_by_Region_1950-2050.png.

5 World Health Organization: WHO Study on global AGEing and adult health (SAGE). http://www.who.int/ healthinfo/sage/en/ (accessed November 5, 2012).

6 Reinhard M, Poulsen PL, Christensen KL: Very poor agreement between routine outpatient clinic office and ambulatory blood pressure: time to improve an old hospital outpatient clinic routine? Blood Press Monit 2016;21:340-344.

7 Cohen JB, Cohen DL: Integrating out-of-office blood pressure in the diagnosis and management of hypertension. Curr Cardiol Rep 2016;18:112.

8 Ntineri A, Stergiou GS, Thijs L, et al: Relationship between office and home blood pressure with increasing age: The International Database of HOme blood pressure in relation to Cardiovascular Outcome (IDHOCO). Hypertens Res 2016;39:612-617.

9 de la Sierra A, Banegas JR, Divison JA, et al: Ambulatory blood pressure in hypertensive patients with inclusion criteria for the SPRINT trial. J Am Soc Hypertens 2016;10:947-953.e5.

10 Wang YC, Shimbo D, Muntner P, Moran AE, Krakoff LR, Schwartz JE: Prevalence of masked hypertension among US adults with nonelevated clinic blood pressure. Am J Epidemiol 2017;185:194-202.

11 Islam MS: Ambulatory blood pressure monitoring in the diagnosis and treatment of hypertension. Adv Exp Med Biol 2017;956:109-116.

12 Etyang AO, Warne B, Kapesa S, et al: Clinical and epidemiological implications of 24-hour ambulatory blood pressure monitoring for the diagnosis of hypertension in Kenyan adults: a population-based study. J Am Heart Assoc 2016;5:e004797.

13 Rhee MY, Kim SW, Choi EH, et al: Prevalence of masked hypertension: a population-based survey in a large city by using 24-hour ambulatory blood pressure monitoring. Korean Circ J 2016;46:681-687.

14 Ruzicka M, Akbari A, Bruketa E, Kayibanda JF, Baril C, Hiremath S: How accurate are home blood pressure devices in use? A cross-sectional study. PLoS One 2016;11:e0155677.

15 Kang YY, Li Y, Huang QF, et al: Accuracy of home versus ambulatory blood pressure monitoring in the diagnosis of white-coat and masked hypertension. J Hypertens 2015;33:1580-1587.

16 Goldberg EM, Levy PD: New approaches to evaluating and monitoring blood pressure. Curr Hypertens Rep 2016;18:49.

17 Landis JR, Koch GG: The measurement of observer agreement for categorical data. Biometrics 1977;33:159174.

18 Schoot TS, Weenk M, van de Belt TH, Engelen LJ, van Goor H, Bredie SJ: A new cuffless device for measuring blood pressure: a real-life validation study. J Med Internet Res 2016;18:e85.

19 IEEE Standards Association: IEEE Std 1708-2014 - IEEE Standard for Wearable Cuffless Blood Pressure Measuring Devices. https://standards.ieee.org/findstds/standard/1708-2014.html.

20 Margolis KL, Asche SE, Bergdall AR, et al: Effect of home blood pressure telemonitoring and pharmacist management on blood pressure control: a cluster randomized clinical trial. JAMA 2013;310:46-56. 